\title{
Assessment of testing methodologies for thin-film vacuum MEMS packages
}

\author{
Qian Li · Hans Goosen · Fred van Keulen · \\ Joost van Beek · Guoqi Zhang
}

Received: 1 January 2008/ Accepted: 30 May 2008/Published online: 3 July 2008

(C) The Author(s) 2008

\begin{abstract}
In this paper, a summary of the most relevant failure mechanisms of thin-film vacuum microelectromechanical systems (MEMS) packages and existing testing techniques will be presented. Then, based on analytical models for thin-film vacuum MEMS packages (volume in the order of $10 \mathrm{E}-111$ ), a feasibility study on options for thin-film vacuum MEMS package testing will be presented. This feasibility study leads to new insights and suggestions for future thin-film vacuum MEMS package testing.
\end{abstract}

\section{Introduction}

Microelectromechanical systems (MEMS) have been investigated for years, but few commercial devices are on the market. Packaging technology is one of the vital limitations. Unlike IC packages, there is no standard process recipe as different material selections, structural designs and processes are needed in MEMS packaging (Chen et al. 2002; Gilleo 2005; Hsu 2004; Rebeiz 2003). Moreover, most MEMS packages not only protect the MEMS device from the operational environment, but also form an integral

Q. Li $(\bowtie)$

Materials Innovation Institute M2i, Mekelweg 2,

2600 GA Delft, The Netherlands

e-mail: q.li@m2i.nl; q.li@tudelft.nl

Q. Li · H. Goosen · F. van Keulen · G. Zhang

Department of Precision and Microsystems Engineering,

Delft University of Technology, Mekelweg 2,

2628 CD Delft, The Netherlands

J. van Beek · G. Zhang

NXP Semiconductors, 5656 AE Eindhoven, The Netherlands part of the microsystems. A typical example is a thin-film vacuum MEMS package; it requires the package to be made of thin-film materials and retain vacuum inside the package to ensure the functional requirements of the device.

\subsection{Thin-film vacuum MEMS packages}

Thin-film packaging aims to fabricate the micro-cavity on the MEMS for wafer-level packaging using micromachining technology. The MEMS is encapsulated with thin film materials. Sacrificial layer etching and deposition techniques are often used to create a package around the MEMS device. Figure 1 shows a conceptual flow of the thin-film package fabrication. This kind of package offers many advantages. First, the typical size of a thin-film package is in the order of microns to nanometers, which fits the trend of miniaturization of the electrical products. Second, the thin-film package needs less space on the wafer, greatly reducing the cost. Third, most thin-film packaging is MEMS fabrication compatible, and, consequently, can be integrated in the backend of the MEMS process. Thus, it allows flexibility in design and is capable of eliminating contamination from the outside environment.

In this paper we will focus on the thin-film vacuum packages. Vacuum inside the package is required for proper performance of many types of MEMS. One typical application is the MEMS resonator, since vacuum is required for a high quality $(Q)$ factor and stable resonant frequency. The vacuum inside the package is normally realized by sealing the package in a vacuum environment. There are two critical concerns for this package. First, it must guarantee a sufficiently high vacuum after the sealing step. Second, it must remain hermetic and vacuum during device operation. 
Fig. 1 A conceptual process flow for creating a thin film package around a MEMS device

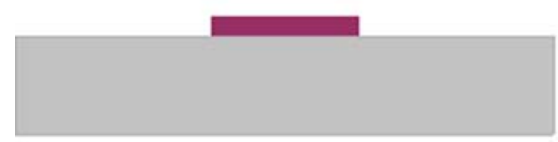

(a) MEMS structure fabrication.

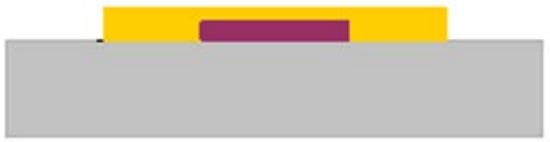

(b) Sacrificial layer deposition above MEMS

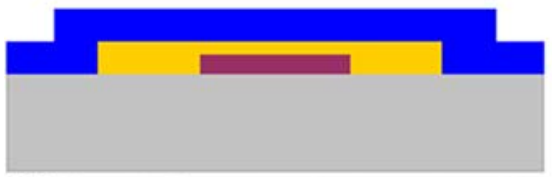

(c) First thin film packaging layer deposition.

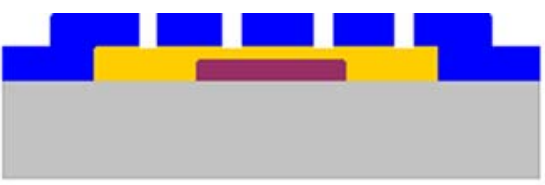

(d) Etching holes on the first thin film packaging layer.

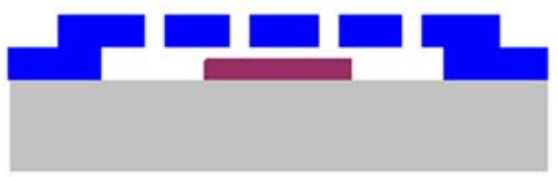

(e) Releasing the sacrificial layer.

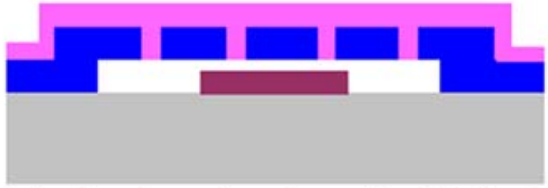

(f) Sealing the package by another thin film layer.

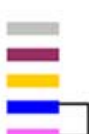

Substrate

MEMS

Sacrificial layer

Thin-film package layers

\subsection{Package parameters}

The thin-film vacuum package investigated in this paper is made of plasma enhanced chemical vapor deposition (PECVD) silicon nitride which has a small volume (Table 1) and is to be designed for a working period of 10 years. After processing, the pressure inside the package is assumed to be 0 bar. The highest permitted pressure is 1 mbar.

\section{Reliability of thin-film vacuum MEMS packages}

Reliability of thin-film vacuum MEMS packages is a major concern for the application of such packaging technology in industry. Many types of failures may happen during processing, assembly, qualification testing and while in operation. Typical failure modes are leaking of the package, outgassing of the package material, collapse of the package capping layer, etc. (Fig. 2). The presence of microcracks in the capping layer can cause leakage, which may be avoided by using thin-films with low residual stress, high young's modulus and a coefficient of thermal expansion (CTE) close to that of the substrate. The process can leave gases inside the thin-film layer which may lead to outgassing under certain conditions (e.g. under a high temperature or after a long period of time) and, consequently, lead to a pressure increase inside the package. The collapse of the capping layer could cause the failure of the package and even the entire device structure. All of these failures will break the vacuum environment inside the MEMS package and cause the failure of the MEMS.

\section{Testing methodologies for thin-film vacuum MEMS packages}

To ensure reliability, testing is of utmost importance. However, testing of a thin-film vacuum MEMS package is complicated and challenging. Due to the small dimension of the thin-film package, the signal to be tested is extremely small; therefore the existing testing techniques may not be readily applied to the small volume MEMS packages. Consequently, testing of MEMS packages requires innovative and specific testing strategies, equipments and methodologies. In this study we will summarize the potential testing techniques for the thin-film vacuum packages, analyze their feasibility for our model and try to present some solutions.

Table 1 Thin-film vacuum package parameters

\begin{tabular}{lllllll}
\hline $\begin{array}{l}\text { Length }(L) \\
(\mu \mathrm{m})\end{array}$ & $\begin{array}{l}\text { Width }(W) \\
(\mu \mathrm{m})\end{array}$ & $\begin{array}{l}\text { Height }(H) \\
(\mu \mathrm{m})\end{array}$ & $\begin{array}{l}\text { Young's modulus }(E) \\
(G P a)\end{array}$ & $\begin{array}{l}\text { Poison's ratio } \\
(v)\end{array}$ & $\begin{array}{l}\text { Highest permitted pressure } \\
(\mathrm{mbar})\end{array}$ & $\begin{array}{l}\text { Life time } \\
(\text { year })\end{array}$ \\
\hline 100 & 100 & 2.5 & 145 & 0.3 & 1 & 10 \\
\hline
\end{tabular}



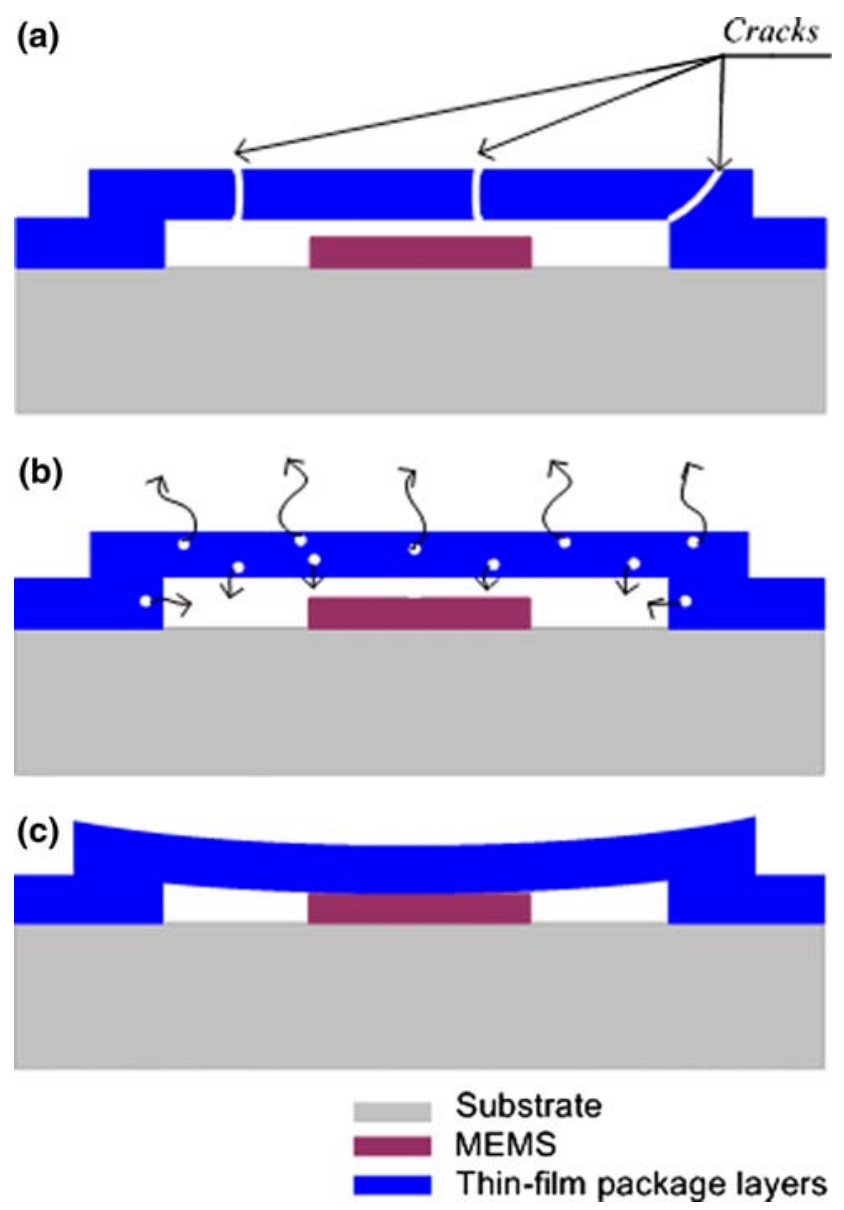

Fig. 2 a Leaking, b outgassing, c collapse of the MEMS package

The testing methodologies for thin-film vacuum packages are divided into two groups. The first category relates to external testing; another involves testing by integrated devices.

External testing is the method to check the vacuum level or hermeticity of the thin-film vacuum package by external testing techniques. Leak test and deflection test are the representative methods from this category and will be discussed in detail.

Testing techniques in the second category make use of integrated devices to measure the vacuum level and the leak rate of the thin-film vacuum package. Often used structures include pressure sensors, moisture sensors, MEMS resonators and accelerometers, etc. Here we will focus on pressure sensors and MEMS resonators, which are often used in research and industry.

\subsection{Leak test}

The purpose of the leak test is to determine the hermeticity of the seal of microelectronic and semiconductor devices with designed internal cavities (MIL-STD-883E 1996).

The method of a leak test is to place the package in a pressurized gas for a certain time, then trace the gas leaking out of the package in a vacuum chamber. Otherwise immerse the package in a certain fluid, and then trace the fluid in some other fluid, which indicates leakage of the package. Often MIL-STD-883 is used as the criteria for leak testing.

A leak is characterized by the leak rate. There are socalled standard leak rate $L$ and measured leak rate $R$. The standard leak rate is defined as that quantity of dry air at $25^{\circ} \mathrm{C}$ flowing through a leak or multiple leak paths per second when the high-pressure side is at $1 \mathrm{~atm}$. (Gillot et al. 2005) (Eq. 1). Measured leak rate $R$ is defined as the leak rate of a given package as measured under specific conditions and employing a specified test medium. The relation between $R$ and $L$ is shown in Eq. 2.

$L=\frac{\Delta P V}{t}$

where $L$ is the standard leak rate in air; $\Delta P$ is the pressure change inside the package; $t$ is the time for the pressure change in seconds; $V$ is the internal volume of the device package cavity.

$R=\alpha L\left(1-e^{\beta L}\right) e^{\gamma L}$

with

$\alpha=\frac{P_{E}}{P_{0}}\left(\frac{M_{A}}{M}\right)^{\frac{1}{2}}, \beta=-\left[\frac{t_{1}}{V P_{0}}\left(\frac{M_{A}}{M}\right)^{\frac{1}{2}}\right], \gamma=-\left[\frac{t_{2}}{V P_{0}}\left(\frac{M_{A}}{M}\right)^{\frac{1}{2}}\right]$

where $R$ is the measured leak rate of tracer gas through the leak; $L$ is the equivalent standard leak rate; $P_{E}$ is the pressure of exposure; $P_{0}$ is the atmospheric pressure; $M_{A}$ is the molecular weight of air in grams; $M$ is the molecular weight of the tracer gas; $t_{1}$ is the time of exposure to $P_{E} ; t_{2}$ is the dwell time between release of pressure and leak detection; $V$ is the internal volume of the device package cavity.

Typically, depending on the covered leak range, the whole leak rate spectrum (Fig. 3) is divided into three regimes: the fine leak regime, the undefined regime and the gross leak regime. The gross leak refers to the measured

Fig. 3 Leak rate spectrum

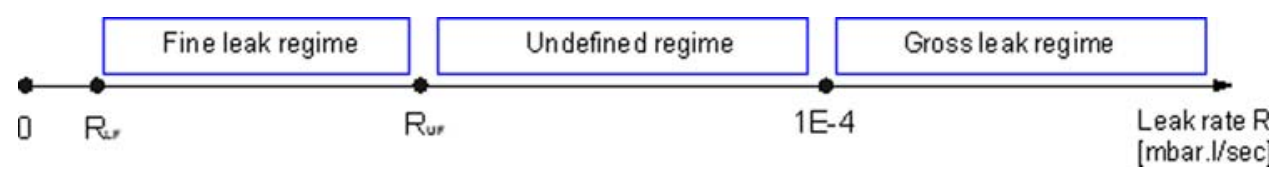


leak rates $R$ larger than a certain value, which is about 1E$4 \mathrm{mbar}$ 1/s. Whereas, fine leak refers to the low leak rate regime; the measured leak rate is larger than the minimal detectable leak rate $R_{L F}$, which is given by the leak detector and smaller than $R_{U F}$, which is decided by the package volume (Jourdain et al. 2002). The undefined regime is the regime between the fine leak and gross leak regime. Bubble gross leak test and helium (He) leak test are the two most frequently used techniques for the gross leak and fine leak regions, respectively, as used for hermeticity testing of the packages.

The advantages of the leak test include:

- There is no need to change the design of the package, since no integrated testing structure is required.

- Side effects due to an additional integrated test structure are eliminated. Time and cost for designing a test structure is saved.

The disadvantages of the leak test include:

- No quantitative vacuum level of the package is known from this test.

- It is not applicable to a very small dimension because of the test sensitivity of the leak detector.

- Normally the cost for the He leak testing is high.

\subsubsection{Bubble gross leak test}

In this method, two liquids with different boiling points are applied. The fluorocarbon liquids (FC-84, boiling point $80^{\circ} \mathrm{C}$ and FC-40, boiling point $161^{\circ} \mathrm{C}$ ) are often used. The package is first placed in the liquid with a lower boiling point for several hours. Then, it is dried and immediately transferred to another liquid and heated to a temperature which is between the boiling points of both liquids. If the first liquid is present inside the package, the bubbles of it will be observed. This methodology is only applicable for the large leaks with leak rate exceeding about $1 \mathrm{E}-$ 4 mbar $1 / \mathrm{s}$.

\subsubsection{Helium leak detect}

He leak testing is a method often used for fine leak detection. This method is according to Method 1014.12, MIL-STD-883G. First, the package is stored in a certain He pressure for some time. Pressurized helium enters the package if there is leakage. Then, the package is placed in a vacuum chamber which is connected to a He mass spectrometer. It is opened and the He which leaked into the package can be detected by the $\mathrm{He}$ mass spectrometer.

For our thin-film vacuum package with a volume of $2.5 \times 10^{-11} 1$, with a highest allowed pressure of $1 \mathrm{mbar}$ and a working time of 10 years, the permitted standard leak rate would be: $L=7.9 \mathrm{E}-20 \mathrm{mbar} 1 / \mathrm{s}$. If $P_{E}=1 \mathrm{bar}$, $t_{1}=2 \mathrm{~h}=7,200 \mathrm{~s}, t_{2}=20 \mathrm{~s}$, the equivalent measured leak rate in $\mathrm{He} R_{1}=1.27 \mathrm{E}-26 \mathrm{mbar} 1 / \mathrm{s}$.

Our highest permitted measured leak rate in $\mathrm{He}$ of $1.27 \mathrm{E}-26 \mathrm{mbar} 1 / \mathrm{s}$ is much lower than the detectable leak rate of the $\mathrm{He}$ leak detector $\left[R_{L F}=4 \mathrm{E}-10 \mathrm{mbar} 1 / \mathrm{s}\right.$ (Jourdain et al. 2002)]. So the above two testing methodologies are not applicable to our thin-film vacuum MEMS package with a volume of $2.5 \times 10^{-11} 1$. The solution is to create a larger volume package or to measure several packages together (Kim et al. 2005).

\subsection{Deflection test}

In this method the pressure inside the package is derived from the deflection of the thin-film package capping layer. Different pressures in and outside the package may result in a deflection of the capping layer (Fig. 4). From the deflection under a known pressure, we can calculate the pressure inside the package.

Following is the theory for the plate deflection which shows the relation between the maximum deflection and the pressure. Here we will only present the theory for the simply supported rectangular capping, valid for an isotropic homogeneous material as an example (Timoshenko and Woinowsky-Krieger 1970; Ugural 1981; Ventsel and Krauthammer 2001).

The maximum deflection of the layer

$\omega_{\max }=\frac{5 P_{\text {total }} l^{4} w^{4}}{384 D\left(l^{4}+w^{4}\right)}$

where $\omega_{\max }$ is the maximum deflection of the plate; $l$ is the length of the plate; $w$ is the width of the plate; $P_{\text {total }}$ is the total pressure on the layer; $D$ is the flexural rigidity of the plate.

With

$D=\frac{E h^{3}}{12\left(1-v^{2}\right)}$

where $h$ is the thickness of the plate; $E$ is the young's modulus; $v$ is the Poisson's ratio.

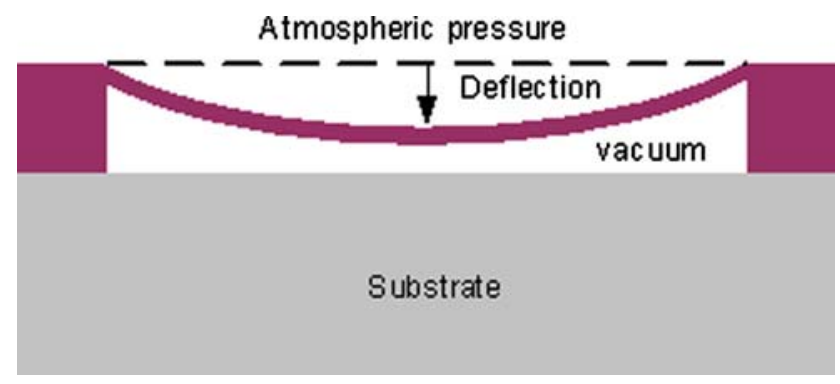

Fig. 4 The hermetically sealed microcap in vacuum is deformed under an ambient environment 
$\omega_{\max }=\frac{5 \Delta P l^{4} w^{4}\left(1-v^{2}\right)}{32 E h^{3}\left(l^{4}+w^{4}\right)}$

$P_{\text {total }}=\frac{32}{5} \frac{E h^{3}}{\left(1-v^{2}\right)} \frac{\left(l^{4}+w^{4}\right)}{l^{4} w^{4}} \omega_{\max }$

$\Delta P_{\text {total }}=\frac{32}{5} \frac{E h^{3}}{\left(1-v^{2}\right)} \frac{\left(l^{4}+w^{4}\right)}{l^{4} w^{4}} \Delta \omega_{\max }$

From Eq. 6, it shows that the absolute pressure on the plate is proportional to the material property, the geometry of the plate and the change of its central deflection. With the known material property, geometry, central deflection and outside pressure, the vacuum inside the package can be derived. The first two parts are fixed when the plate is designed and fabricated. The pressure difference is only determined by the change of the central deflection (see Eq. 7). The accuracy of the pressure measurement inside package depends on the accuracy of the deflection measurement.

The advantages of the deflection test include:

- The pressure inside the package is easily calculated from the formula if the deflection is measured. Thus not only the hermeticity but also the quantitative vacuum level of the package is measured.

- The test is simple in principle. The deflection measurement can be easily done by contact or optical profile measurement equipments.

The disadvantages of the deflection test include:

- The capping for the MEMS package may include several materials and some irregular structures. In this case, the pressure inside the package is not easily derived from deflection of the capping.

- In the capping layer, residual stress may cause unpredictable shape changes of the package. These effects are normally not well understood and often happen randomly for different devices. So the equation for the deflection versus pressure may not be valid if this is the case.

- Some profile measurements may introduce extra stresses on the package, making the pressure prediction less accurate.

Applying Eq. 7 to our package (with parameters in Table 1), when its pressure increases from 0 to $1 \mathrm{mbar}$, the change of the deflection of the capping layer can be derived: $\Delta \omega=3 \mathrm{E}-7 \mathrm{~m}$.

The highest permitted deflection change of our packaging layer in its life time (10 years) is $3 \mathrm{E}-7 \mathrm{~m}$. Assume that the deflection of the layer changes gradually at the same rate, the deflection change of the packaging layer in every week can be estimated to be $\Delta \omega /(10 \times 12 \times 4)=6.25 \mathrm{E}-$ $10 \mathrm{~m} /$ week. This deflection change per week is several angstroms, which is near the highest resolution of most existing profile measurement equipments. For instance, the resolution of one optical profiler Wyko NT3300, which is often used for MEMS measurement, is about $1 \AA$. The above testing methodology is not easily applied but still possible for our thin-film vacuum MEMS package with a small volume.

\subsection{Integrated pressure sensor}

For the high vacuum measurement of the package, a MEMS Pirani gauge is often used as the pressure sensor because of its micron size and it is easily encapsulated in the thin-film package. Moreover, the Pirani gauge can not only check the hermeticity but also detect the quantitative vacuum level of the package (Chae et al. 2005; Chou et al. 1995; Chou and Shie 1997; de Jong et al. 2003; Mastrangelo and Muller 1991; Zhang et al. 2006).

A Pirani gauge (Fig. 5) consists of a sensor wire, which is in contact with the pressure to be measured. The operation principle of the Pirani gauge is that the temperaturedependent resistance of the gauge is dependent on the ambient pressure, since a large part of the heat generated by Joule heating in the gauge is transferred through the air to the substrate (gaseous conduction) (Mastrangelo 1991).

The thermal impedance TI, which is defined by the Eq. 8, is dependent on the pressure. From the calibrated measurement of TI versus pressure, the pressure inside package is known if the thermal impedance is known. Figure 6 shows an example of a measured relation between the thermal impedance and pressure (He and Kim 2007).

$\mathrm{TI}=\frac{\Delta T_{\text {avg }}}{\Delta P_{E}}$

where $\Delta T_{\text {avg }}$ is the change of the average temperature across the Pirani gauge; $\Delta P_{E}$ is the change of the electrical power.

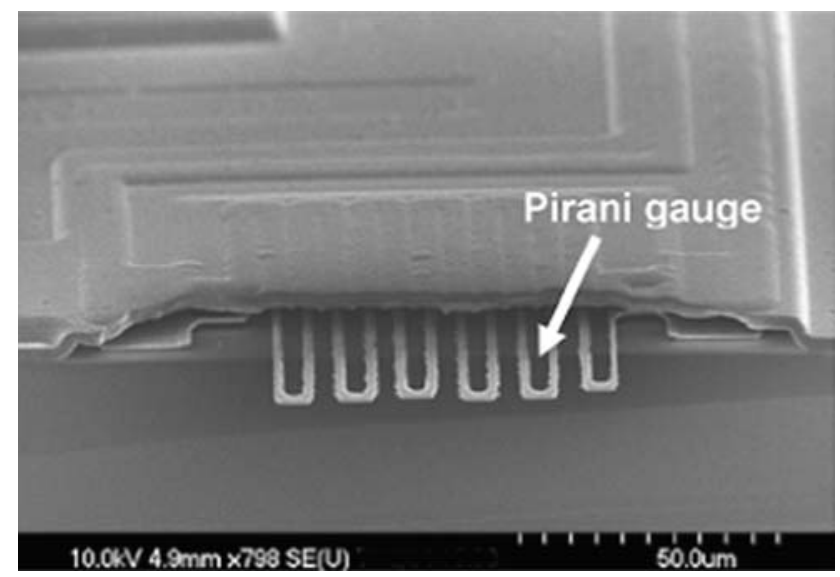

Fig. 5 A SEM picture of a polysilicon Pirani gauge encapsulated by porous polysilicon shell and sealed [reprinting with author's permission] 


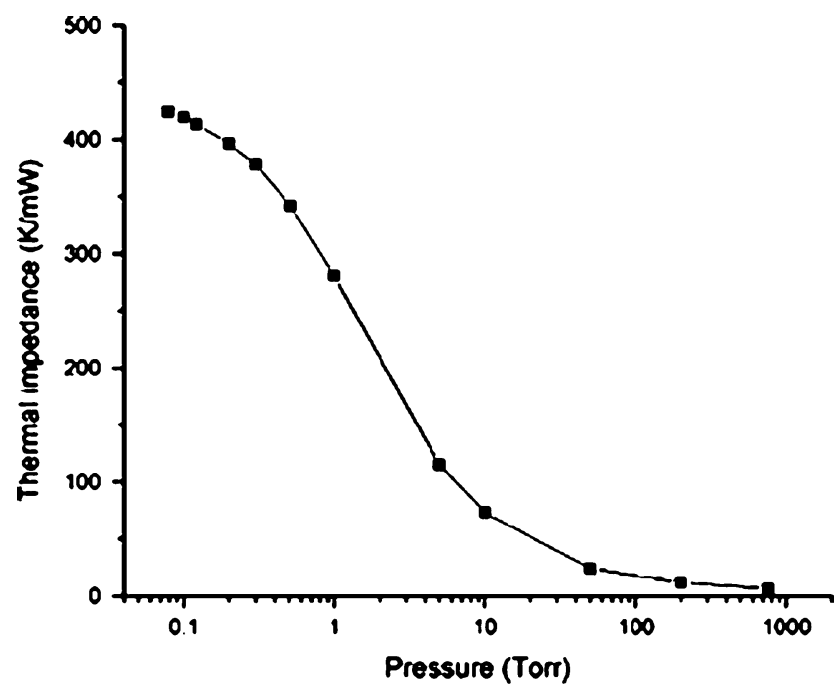

Fig. 6 Thermal impedance of a Pirani gauge at different pressure [reprinting with author's permission]

For a bridge Pirani gauge, the high and low detectable pressure limits of the linear pressure range, $P_{h}$ and $P_{l}$, respectively, are given as follows (He and Kim 2007):

$P_{h}=\frac{2 \eta k_{g}(\infty) T_{s} \omega}{\pi \alpha_{E} \bar{v} s d}$

$P_{l}=\alpha_{E}\left(\frac{k_{b}}{\eta k_{g}(\infty)}\right)\left(\frac{s z}{l^{2}}\right) P_{h}$

where $\eta$ is the excess-flux coefficient, which accounts for the fringing heat flux of the bridge element and can be obtained analytically; $k_{g}(\infty)$ is the thermal conductivity of the gas at atmospheric pressure; $T_{s}$ is the substrate temperature; $\alpha_{E}$ is the thermal-accommodation coefficient; $\bar{v}$ is the average gas molecular velocity; $d$ is the microbridge perimeter; $k_{b}$ is the thermal conductivity of the bridge material; $s$ is the distance above the substrate of the microbridge; $\omega$ is the width of the microbridge; $z$ is the thickness of the microbridge; $l$ is the length of the microbridge.

Typically, the pressure detection range of the Pirani gauge is $10 \mathrm{E}-3$ to $30 \mathrm{mbar}$ (VG Scienta 2003). The detection range can be adjusted by changing the dimensions of the bridge as well as the gap between the bridge and the substrate.

The pressure of our thin-film package should be lower than 1 mbar, which is well within the working range of the Pirani gauge. Thus, the Pirani gauge can be used to measure the package's pressure. However, the following issues must be considered and carefully analyzed when applying this testing method for thin-film package characterization. First, since the pressure to be detected by the Pirani gauge is derived from the thermal conductivity of the ambient gas and thermal conductivities of different gases are not same, calibration measurement must be done for different gas composition, which may make the test more complicated and may include errors. Second, the gas byproducts of the MEMS fabrication and outgassing of the thin-film package layer also need to be considered as a part of the ambient gases for the calibration measurement, but in most cases the composition and amount of these gases are not easy to detect.

\subsection{MEMS resonator}

In this case, the characteristics of some encapsulated MEMS, which are not designed to be pressure sensors, are used to evaluate the vacuum level of the packages. The performance of these MEMS depends on the quality of the package. For example, the $Q$-factor of a MEMS resonator depends on the pressure inside the package within a certain pressure range (Blom et al. 1992; Lee et al. 2003). Thus, the pressure inside a package can be determined if we know the $Q$-factor of the device.

The relation between $Q$-factor and pressure is obtained by measuring an unpackaged resonator in a vacuum chamber with a changing vacuum level. When the $Q$-factor of the vacuum packaged resonator is measured, the inside pressure of the package device is estimated by the $Q$ versus $P$ curve. Figure 7 shows an example of the relation between $Q$-factor and ambient pressure. For a thin-film packaged resonator, which has a $Q$-factor of 1,818 (Fig. 8), the pressure inside this package is estimated to be $80 \mathrm{mbar}$ according to this $Q$ versus $P$ curve, assuming the gas inside the package is the same as the gas used for the calibration measurement.

The advantages of the testing by MEMS resonators include:

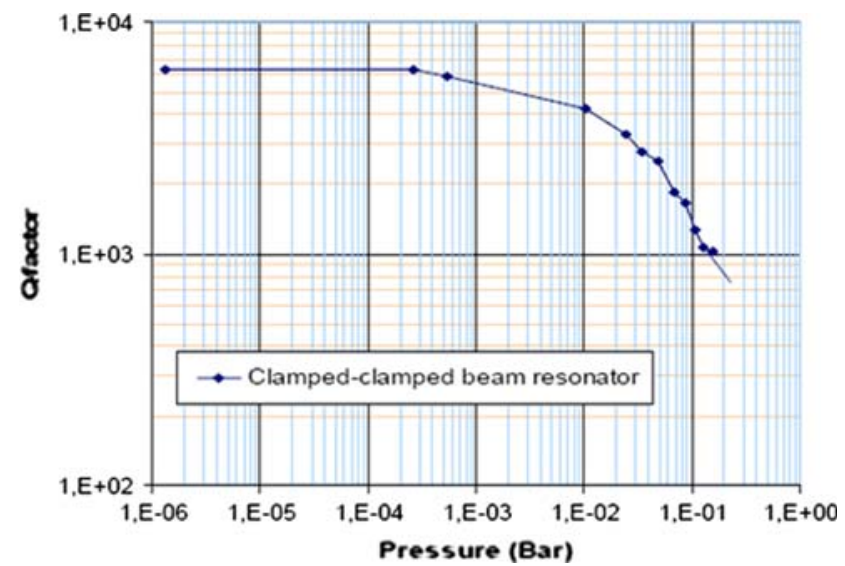

Fig. 7 The $Q$-factor versus vacuum pressure for an unpackaged resonator in a vacuum chamber (pressure changed by nitrogen) 
Fig. 8 Measured (blue dots) and fitted (red line) data for the magnitude of the $\mathrm{Y}$ parameter (a), phase angle of the $\mathrm{Y}$ parameter (b)
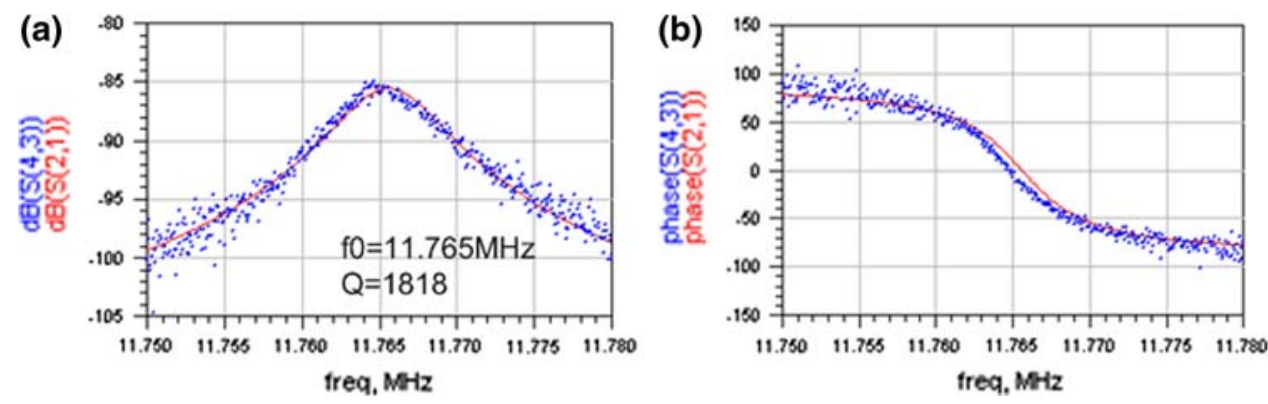

- The fabrication of the MEMS resonator can be thin-film packaging process compatible.

- A quantitative vacuum level can be determined.

The disadvantages of the resonator test include:

- This method is only valid for a small pressure range (Blom et al. 1992). At high vacuum and higher pressure range, the $Q$-factor does not depend on ambient pressure but on other parameters.

For our package, when its pressure is in the high vacuum regime, this test method will not work, since the $Q$-factor is not pressure dependent at this pressure regime; when the package has the pressure in the medium to low vacuum regime, this test method may work.

\section{Conclusions}

In this paper, testing methodologies for thin-film vacuum MEMS packages are divided into two categories: external testing and in situ testing. The four most promising testing methodologies, viz., the leak test, deflection test, Pirani gauge and MEMS resonator, are selected and summarized. From the feasibility study on each option for our package, it is shown that He leak test is not applicable since the resolution of this technique is insufficient for the leakage of the thin-film package. The other three, deflection test, Pirani gauge and MEMS resonator may be used for thin-film package testing. However, the deflection test is not easily applied to the real thin-film package because of the thermal mismatch between materials or unknown residual stress in the layer may cause stiffening or buckling of the capping layer. So the most promising testing methods are the Pirani gauge for high vacuum and MEMS resonator for low vacuum measurement. However, both methods require calibration for accurate quantitative results.

Acknowledgments This research was carried out under the project number MC3.05230 in the framework of the Research Program of the Materials innovation institute M2i (http://www.m2i.nl), the former Netherlands Institute for Metals Research.
Open Access This article is distributed under the terms of the Creative Commons Attribution Noncommercial License which permits any noncommercial use, distribution, and reproduction in any medium, provided the original author(s) and source are credited.

\section{References}

Blom FR, Bouwstra S, Elwenspoek M, Fluitman JHJ (1992) Dependence of the quality factor of micromachined silicon beam resonators on pressure and geometry. J Vac Sci Technol B 10(1):19-26. doi:10.1116/1.586300

Chae J, Stark BH, Najafi K (2005) A micromachined Pirani gauge with dual heat sinks. IEEE Tran Adv Packag 28(4):619-625. doi: 10.1109/TADVP.2005.858316

Chen J-Y, Huang L-S, Chu C-H, Chang P (2002) A new transferred ultra-thin silicon micropackaging. J Micromech Microeng 12:406-409. doi:10.1088/0960-1317/12/4/310

Chou BCS, Shie J-S (1997) An innovative Pirani pressure sensor. In: Proceedings of international conference on solid-state sensors and actuators, pp 1465-1468

Chou BCS, Shie J-S, Clien Y-M (1995) A highly sensitive Pirani vacuum gauge. In: Proceedings of the 8th international conference on solid-state sensors and actuators, and Eurosensors IX, pp $167-170$

Cianci E, Foglietti V, Coppa A (2006) Young's modulus and residual stress of DF PECVD silicon nitride for MEMS free standing membranes. In: Proceedings of 32nd international conference on micro- and nano-engineering, P-MST39-microsystems and their fabrication

de Jong BR, Bula WP, Zalewski D, van Baar JJ, Wiegerink RJ (2003) Pirani pressure sensor with distributed temperature measurement. In: Proceedings of IEEE conference on sensors, pp 718722

Gilleo K (2005) MEMS/MOEMS packaging, concepts, designs, materials, and processes. McGraw-Hill, New York

Gillot C, Lagoutte E, Charvet PL, Souchon F, Sillon N (2005) Wafer level thin film encapsulation for MEMS. In: Proceedings of conference on high density microsystem design and packaging and component failure analysis, pp 1-4

He RH, Kim C-J (2007) On-wafer monolithic encapsulation by surface micromachining with porous polysilicon shell. J Microelectromech Syst 16(2):462-472. doi:10.1109/JMEMS.2007. 892797

Hsu T-R (2004) MEMS packaging, INSPEC

Jourdain A, De Moor P, Pamidighantam S, Tilmans HAC (2002) Investigation of the hermeticity of BCB-sealed cavities for housing (RF-)MEMS devices. In: Proceedings of the fifteenth IEEE international conference micro electro mechanical systems, pp 677-680 
Kim W, Wang Q, Jung K, Hwang J, Lee M, Jung K, Ham SJ, Moon C (2005) A low temperature, hermetic wafer level packaging method for RF MEMS switch. In: Proceedings of 2005 electronic components and technology conference, pp 11031108

Lee B, Seok S, Chun KK (2003) A study on wafer level vacuum packaging for MEMS devices. J Micromech Microeng 13:663669. doi:10.1088/0960-1317/13/5/318

Mastrangelo CH (1991) Thermal applications of microbridges. PhD Thesis, University of California, Berkeley

Mastrangelo CH, Muller RS (1991) Microfabricated thermal absolutepressure sensor with on-chip digital front-end processor. J Solid State Circuits 26(12):1998-2007. doi:10.1109/4.104194

MIL-STD-883E (1996) Test method standard microcircuits. Department of Defense, USA
Rebeiz GM (2003) RF MEMS theory, design, and technology. Wiley, London

VG Scienta (2003) Pirani gauge-introduction. http://www. thermovacgen.com. Accessed 21 May 2008

Timoshenko SP, Woinowsky-Krieger S (1970) Theory of plates and shells. McGraw-Hill, New York

Ugural A (1981) Stress in plates and shells. McGraw-Hill, New York, pp 80-83

Ventsel E, Krauthammer T (2001) Thin plates and shells, theory, analysis, and applications. CRC Press, West Palm Beach

Zhang FT, Tang Z, Yu J, Jin RC (2006) A micro-Pirani vacuum gauge based on micro-hotplate technology. J Sens Actuators A Phys 126(2):300-305 